Results after surgical treatment of liver metastases in patients with high-grade gastroenteropancreatic neuroendocrine carcinomas

Galleberg, R. B.

2017-09

Galleberg , R B , Knigge , U , Janson, E T , Vestermark , L W , Haugvik , S -P , Ladekarl , M , Langer , S W , Gronbaek, H, Osterlund, P , Hjortland, G O , Assmus , J, Tang , L , Perren , A \& Sorbye , H 2017 , ' Results after surgical treatment of liver metastases in patients with high-grade gastroenteropancreatic neuroendocrine carcinomas ' , European Journal of Surgical Oncology , vol. 43 , no. 9 , pp. 1682-1689 . https://doi.org/10.1016/j.ejso.2017.04.010

http://hdl.handle.net/10138/297993

https://doi.org/10.1016/j.ejso.2017.04.010

publishedVersion

Downloaded from Helda, University of Helsinki institutional repository.

This is an electronic reprint of the original article.

This reprint may differ from the original in pagination and typographic detail.

Please cite the original version. 


\title{
Results after surgical treatment of liver metastases in patients with high-grade gastroenteropancreatic neuroendocrine carcinomas
}

\author{
R.B. Galleberg ${ }^{\text {a,* }}$, U. Knigge ${ }^{\mathrm{b}}$, E. Tiensuu Janson ${ }^{\mathrm{c}}$, \\ L.W. Vestermark ${ }^{d}$, S.-P. Haugvik ${ }^{\mathrm{e}}$, M. Ladekarl $^{\mathrm{f}}$, S.W. Langer ${ }^{\mathrm{g}}$, \\ H. Grønbæk ${ }^{\text {h }}$, P. Österlund ${ }^{\mathrm{i}}$, G.O. Hjortland ${ }^{\mathrm{j}}$, J. Assmus ${ }^{\mathrm{k}}$, L. Tang ${ }^{1}$, \\ A. Perren ${ }^{\mathrm{m}}$, H. Sorbye ${ }^{\mathrm{a}, \mathrm{n}}$ \\ ${ }^{a}$ Department of Oncology, Haukeland University Hospital, Bergen, Norway \\ ${ }^{\mathrm{b}}$ Departments of Surgery C and Endocrinology PE, Rigshospitalet, University of Copenhagen, Denmark \\ ${ }^{\mathrm{c}}$ Department of Medical Sciences, Uppsala University, Sweden \\ ${ }^{\mathrm{d}}$ Department of Oncology, Odense University Hospital, Denmark \\ ${ }^{\mathrm{e}}$ Department of Hepato-Pancreato-Biliary Surgery, Rikshospitalet, Oslo University Hospital, Norway \\ ${ }^{\mathrm{f}}$ Department of Oncology, Aarhus University Hospital, Denmark \\ ${ }^{\mathrm{g}}$ Department of Oncology, Rigshospitalet, University of Copenhagen, Denmark \\ ${ }^{\mathrm{h}}$ Department of Hepatology and Gastroenterology, Aarhus University Hospital, Denmark \\ ${ }^{\mathrm{i}}$ Department of Oncology, Helsinki University Central Hospital, Finland \\ ${ }^{\mathrm{j}}$ Department of Oncology, Oslo University Hospital, Norway \\ ${ }^{\mathrm{k}}$ Center for Clinical Research, Haukeland University Hospital, Bergen, Norway \\ ${ }^{1}$ Department of Pathology, MSKCC, New York, USA \\ ${ }^{\mathrm{m}}$ Department of Pathology, University of Bern, Switzerland \\ ${ }^{\mathrm{n}}$ Department of Clinical Science, University of Bergen, Norway
}

Accepted 21 April 2017

Available online 4 May 2017

\begin{abstract}
Background: Gastroenteropancreatic neuroendocrine carcinomas (GEP-NEC) are generally characterized by synchronous metastases, high aggressiveness and a dismal prognosis. Current international guidelines do not recommend surgical treatment of liver metastases, however the existing data are scarce. The aim of this study was to evaluate the results of curatively intended resection/radiofrequency ablation (RFA) of liver metastases in patients with metastatic GEP-NEC.

Methods: 32 patients with a diagnosis of high-grade gastroenteropancreatic neuroendocrine neoplasm (Ki-67 > 20\%) and with intended curative resection/RFA of liver metastases, were identified among 840 patients from two Nordic GEP-NEC registries. Tumor morphology (well vs poor differentiation) was reassessed. Overall survival (OS) and progression-free survival (PFS) was assessed by Kaplan-Meier analyses for the entire cohort and for subgroups.

Results: Median OS after resection/RFA of liver metastases was 35.9 months (95\%-CI: 20.6-51.3) with a five-year OS of $43 \%$. The median PFS was 8.4 months (95\%-CI: 3.9-13). Four patients (13\%) were disease-free after 5 years. Two patients had well-differentiated morphology (NET G3) and 20 patients (63\%) had Ki-67 $\geq 55 \%$. A Ki-67 < 55\% and receiving adjuvant chemotherapy were statistically significant factors of improved OS after liver resection/RFA.
\end{abstract}

\footnotetext{
* Corresponding author. Department of Oncology, Haukeland University Hospital, 5021 Bergen, Norway. Fax: +47 55973918.

E-mail addresses: renate.berget.galleberg@helse-bergen.no (R.B. Galleberg), rxs484@ku.dk (U. Knigge), eva.tiensuu_janson@medsci.uu.se (E. Tiensuu Janson), lene.vestermark@syd.dk (L.W. Vestermark), sphaugvik@yahoo.de (S.-P. Haugvik), mortlade@rm.dk (M. Ladekarl), swlanger@dadlnet.dk (S.W. Langer), henning.gronbaek@aarhus.rm.dk (H. Grønbæk), pia.osterlund@pshp.fi (P. Österlund), goo@ous-hf.no (G.O. Hjortland), jorg.assmus@helse-bergen.no (J. Assmus), tangl@MSKCC.ORG (L. Tang), aurel.perren@pathology.unibe.ch (A. Perren), Halfdan.sorbye@helse-bergen.no (H. Sorbye).
} 
Conclusion: This study shows a long median and long term survival after liver surgery/RFA for these selected metastatic GEP-NEC patients, particularly for the group with a Ki-67 in the relatively lower G3 range. Our findings indicate a possible role for surgical treatment of liver metastases in the management of this patient population.

(C) 2017 Elsevier Ltd, BASO The Association for Cancer Surgery, and the European Society of Surgical Oncology. All rights reserved.

Keywords: Neuroendocrine carcinoma; Metastases; Liver; Surgery; Survival

\section{Introduction}

Neuroendocrine carcinoma (NEC) is broadly defined as a poorly differentiated neuroendocrine neoplasm (NEN) with Ki-67 $>20 \%$ or mitotic rate $>20 / 10$ high-power fields. ${ }^{1}$ In contrast to well differentiated NENs (WHO G1-G2), NECs (WHO G3) are highly aggressive with a propensity for early metastases and a dismal prognosis. ${ }^{2-6}$ At the time of diagnosis $57-88 \%$ of patients with gastroenteropancreatic NECs (GEP-NEC) have distant disease with the liver as the main metastatic site. ${ }^{2,5-7}$ Patients with localized GEP-NECs have a median survival of 16 months, while for the group with metastases median survival is only 5 months. ${ }^{6}$ Current guidelines recommend platinum-based chemotherapy as first-line treatment in the metastatic setting. ${ }^{8-10}$ However, median survival is only $11-13$ months and three-year survival $5-10 \%$ for these chemotherapy treated patients. ${ }^{5,711}$

Due to the highly aggressive behavior and high risk of metastatic disease, the benefit of surgical treatment for GEP-NEC patients has been questioned. International guidelines currently recommend surgery for NENs G1/G2 with resectable liver metastases and in selected cases as debulking surgery, while for GEP-NECs (WHO G3), surgery in the metastatic setting is not recommended. ${ }^{12-14}$ Published data to support these recommendations regarding GEP-NECs are scarce. Two studies evaluating the role of surgical resection of liver metastases in NEN patients, found a median survival of $6-15$ months for the minor fraction of patients with poorly differentiated NENs. ${ }^{15,16}$ Two case reports describe long-term survival in GEPNEC patients after locoregional treatment of their liver metastases. ${ }^{17,18}$

Thus the possible benefit of liver surgery in metastatic GEP-NECs is unsettled. With our study we aim to further evaluate the possible role of surgical treatment of liver metastases in this patient population.

\section{Patients and methods}

Patients with high-grade gastroenteropancreatic neuroendocrine neoplasm (WHO G3) with surgical treatment of liver metastases were identified from two combined medical/surgical Nordic NEC registries. One registry is a retrospective GEP-NEC database consisting of 485 patients diagnosed between 2000 and 2012. The second registry is a prospective GEP-NEC database of 355 patients, collected from January 2013 to September 2015. Twelve Nordic tertiary care institutions have submitted data. Informed consent has been obtained from all patients, and the study has been approved by the medical ethics committees of all participating countries. The inclusion criteria for the present study were: Histopathological confirmed diagnosis of a high-grade neuroendocrine neoplasm (NEN) with Ki-67 $>20 \%$, a gastroenteropancreatic primary or an unknown primary with predominantly abdominal tumor burden, either synchronous or metachronous liver metastases, surgical resection and/or RFA of metastatic disease in the liver with a curative intent. RFA was accepted as a treatment modality as published data support a role for this approach when treating selected patients with limited size liver metastases from colorectal cancer with a curative intent. ${ }^{19}$ Tumor morphology was classified as small-cell or non-small cell. A central review to assess histological differentiation (well differentiated morphology vs poorly differentiated morphology) was performed by four experienced neuroendocrine pathologists (LT, AP, JYS, BF). If the Ki-67 value was reported from both the primary tumor and metastases, the higher value was adopted. A cut-off value of 55\% for Ki-67 was used when performing statistical analyses. ${ }^{11}$ We chose to use progression-free survival instead of disease-free survival as some of our patients never became disease free. The patient who died within 30 days of surgery was excluded from the subgroup analysis comparing patients that did and did not receive adjuvant chemotherapy. This was in order to avoid potential bias as this patient died before chemotherapy could be given. Previously described prognostic markers in GEPNEC patients were applied for subgroup analyses. ${ }^{11,15} \mathrm{Num}-$ ber of metastases is an important prognostic marker after hepatic surgery for metastatic colorectal cancer, and was therefore included in the analyses. ${ }^{20,21}$

\section{Statistical analyses}

OS was defined as the time from surgical treatment of liver metastases to last follow-up or death. PFS was defined as the time from surgical treatment of liver metastases until progression or recurrence of the disease. Descriptive methods were used to characterize the patient population. The survival was assessed by Kaplan-Meier analyses for the entire patient population and for subgroups (one criteria at a time). We compared the subgroups by logrank and 
Table 1

Patient Characteristics for 32 patients with surgically treated metastatic gastroenteropancreatic neuroendocrine carcinoma.

\begin{tabular}{|c|c|c|}
\hline & $\begin{array}{l}\text { Valid } \\
\text { cases }\end{array}$ & $\begin{array}{l}\text { Number of } \\
\text { patients }(\%)\end{array}$ \\
\hline Total number & 32 & \\
\hline Sex: Male & 32 & $19(59.4 \%)$ \\
\hline Median age (range) & 32 & $55(25-79)$ \\
\hline Performance status (PS) & 32 & \\
\hline PS 0 & & $27(84.4 \%)$ \\
\hline PS 1 & & $4(12.5 \%)$ \\
\hline PS 2 & & $1(3.1 \%)$ \\
\hline Location of Primary Tumor & 32 & \\
\hline Pancreas & & $14(43.8 \%)$ \\
\hline Colon & & $9(28.1 \%)$ \\
\hline Rectum & & $3(9.4 \%)$ \\
\hline Gastric & & $1(3.1 \%)$ \\
\hline Unknown & & $3(9.4 \%)$ \\
\hline Other GI & & $2(6.3 \%)$ \\
\hline $\begin{array}{l}\text { Type of pancreatic primary } \\
\text { tumor resection }\end{array}$ & 14 & \\
\hline Whipple & & $4(28.6 \%)$ \\
\hline Distal pancreas resection & & $7(50 \%)$ \\
\hline Total pancreatectomy & & $2(14.3 \%)$ \\
\hline Not resected & & $1(7.1 \%)$ \\
\hline Ki-67-level & 32 & \\
\hline$\geq 55 \%$ & & $20(62.5 \%)$ \\
\hline $21-54 \%$ & & $12(37.5 \%)$ \\
\hline Morphology & 32 & \\
\hline Small cell & & $7(21.9 \%)$ \\
\hline Non-small cell & & $25(78.1 \%)$ \\
\hline Differentiation & 32 & \\
\hline Poorly differentiated & & $24(75.0 \%)$ \\
\hline Well differentiated & & $2(6.3 \%)$ \\
\hline Inconclusive & & $1(3.1 \%)$ \\
\hline Slides not available for review & & $5(15.6 \%)$ \\
\hline Metastatic presentation & 32 & \\
\hline Synchronous & & $28(87.5 \%)$ \\
\hline Metachronous & & $4(12.5 \%)$ \\
\hline Number of liver metastasis & 30 & \\
\hline 1 & & $13(40.6 \%)$ \\
\hline 2 & & $5(15.6 \%)$ \\
\hline 3 & & $5(15.6 \%)$ \\
\hline$>3$ & & $7(21.9 \%)$ \\
\hline Site of metastasis & 32 & \\
\hline Unilateral & & $24(75.0 \%)$ \\
\hline Bilateral & & $8(25.0 \%)$ \\
\hline Procedure performed & 32 & \\
\hline Left hepatectomi & & $3(9.4 \%)$ \\
\hline Right hepatectomi & & $6(18.8 \%)$ \\
\hline Atypical resection & & $5(15.6 \%)$ \\
\hline 1 segment resected & & $8(25.0 \%)$ \\
\hline 2 segments resected & & $7(21.9 \%)$ \\
\hline RFA combined with surgery & & $3(9.4 \%)$ \\
\hline Isolated RFA & & $6(18.8 \%)$ \\
\hline R-status & 32 & \\
\hline R0 & & $21(65.6 \%)$ \\
\hline $\mathrm{R} 1$ & & $2(6.3 \%)$ \\
\hline $\mathrm{R} 2$ & & $4(12.5 \%)$ \\
\hline NA (after Radiofrequency ablation) & & $5(15.6 \%)$ \\
\hline $\begin{array}{l}\text { Synchronous resection of } \\
\text { the primary tumor }\end{array}$ & 27 & \\
\hline Yes & & $16(59.3 \%)$ \\
\hline No & & $11(40.7 \%)$ \\
\hline Neoadjuvant chemotherapy & 32 & \\
\hline
\end{tabular}

Table 1 (continued)

\begin{tabular}{lll}
\hline & $\begin{array}{c}\text { Valid } \\
\text { cases }\end{array}$ & $\begin{array}{l}\text { Number of } \\
\text { patients (\%) }\end{array}$ \\
\hline Cisplatin/etoposide & & $6(18.8 \%)$ \\
Carboplatin/etoposide & & $1(3.1 \%)$ \\
Temozolomide/capecitabine & & $1(3.1 \%)$ \\
Other medical tumor treatment & & $4(12.5 \%)$ \\
None & 32 & $20(62.5 \%)$ \\
Adjuvant chemotherapy & & $4(12.5 \%)$ \\
Cisplatin/etoposide & & $3(9.4 \%)$ \\
Carboplatin/etoposide & & $1(3.1 \%)$ \\
Temozolomide/capecitabine & $5(15.6 \%)$ \\
Other medical tumor treatment & & $19(59.4 \%)$ \\
None & &
\end{tabular}

Breslow tests. Since the proportional hazard assumption could not be approved we used the Breslow test as main criterion and abstained from fitting a Cox model. The general significance level was set to 0.05. All computation was done using SPSS 22 (IBM Corp., Armonk, NY, 2013) and the graphics were produced by Matlab 7.10 (The MathWorks Inc., Natick, MA, 2010).

\section{Results}

\section{Patient characteristics}

From the combined Nordic GEP-NEC registries of 840 patients, we identified 40 patients who had undergone surgical treatment of their liver metastases. Eight patients were later excluded as they did not fulfill the inclusion criteria: One patient was not operated on with a curative intent; two patients underwent surgery several years before they had a NEC diagnosis; one patient had a primary mixed adenoneuroendocrine carcinoma (MANEC) and an adenocarcinoma removed from the liver; two patients had a Ki$67 \leq 20 \%$ after pathological re-evaluation; one patient had a large cell carcinoma with negative chromogranin A and synaptophysin and, after pathological re-evaluation, did not qualify as NEN. Among the remaining 32 patients, 29 had a known primary site and 27 of these had the primary tumor resected. One patient lacked information regarding surgical status of the primary tumor and one patient had the liver metastases resected before the primary pancreatic tumor was found. This pancreatic tumor was not resected. The patients with unknown primary tumors had the diagnosis made on histology from liver biopsies. One of these three patients underwent a right-sided hemicolectomy due to a highly differentiated adenocarcinoma 2 months after liver surgery. Some of the cells in the resected colon stained positive for $\mathrm{CgA}$ and synaptophysin, and NEC was found in the local lymph nodes. In $21(66 \%)$ of the patients a NEC diagnosis was present before surgery. Distant lymph node involvement was reported for three patients, and these lymph nodes were removed simultaneously with the liver surgery in two of the patients. For the last patient relevant information is missing. Six patients were treated solely 
with RFA. Four of these had liver metastases $<40 \mathrm{~mm}$, the last two with metastasis of $40 \mathrm{~mm}$ and $50 \mathrm{~mm}$. The median interval from the primary NEC diagnosis to the diagnosis of liver metastases was 0 months (range $0-27$ months) as most patients had synchronous disease. From the diagnosis of liver metastases to liver surgery the median interval was 1.5 month (range $0-57$ months). The median interval between primary tumor resection and liver metastases resection/RFA was 0 months (range -2 to 29). The patients were followed up for a median of 38 months (range 1-50 months). For the 12 patients still alive at the time of the analysis, median follow-up was 46 months (range 23-184 months). Table 1 describes the different procedures performed on the liver. The median diameter of the largest liver metastasis was $3.5 \mathrm{~cm}$ (range $1-25 \mathrm{~cm}$ ). Median Ki-67 was $60 \%$ (range $25-100 \%$ ) and $63 \%$ of the patients had Ki$67 \geq 55 \%$. Twenty three patients $(70 \%)$ were amenable for the central pathological review of the morphology. For three of the remaining patients the slides were evaluated by one of the study pathologists, these three had Ki-67 values $>80 \%$ and were all deemed poorly differentiated. In one patient, with a Ki-67 of $60 \%$, the degree of differentiation could not be determined as the specimen was suboptimal. For the remaining five patients the histological slides could not be located. These had Ki-67 values of 30\%, 37\%, $45 \%, 70 \%$ and $95 \%$. A complete list of patient characteristics is presented in Table 1.

\section{Overall survival}

No patients were lost to follow-up. The median overall survival after liver surgery/RFA was 35.9 months (95\% CI 20.6-51.3) (Fig. 1a). Three-year survival after surgery/RFA was $47 \%$ and 5-year survival $43 \%$ (Table 2). Patients with a Ki-67 of $21-54 \%$ had significantly longer survival than the group with a higher Ki-67 (61.6 vs. 21.2 months $\mathrm{P}=0.018$ ) (Fig. 1b). There was a trend towards longer survival for the group with a pancreatic primary, however these results did not reach statistical significance (40.6 vs. 21.2 months $\mathrm{P}=0.146$ ) (Fig. 1c). There was a statistically significant longer survival for the group receiving adjuvant chemotherapy $(66.8$ vs. 29.9 months $\mathrm{P}=0.028$ ) (Fig. 1d). No significant difference was found when comparing the groups that did and did not receive neoadjuvant chemotherapy $(>29.9 \quad$ vs. 35.7 months $\mathrm{P}=0.142$ ) (Table 2). Only two of the 26 patients with a known differentiation status had well differentiated tumor morphology (NET G3), these two had an OS

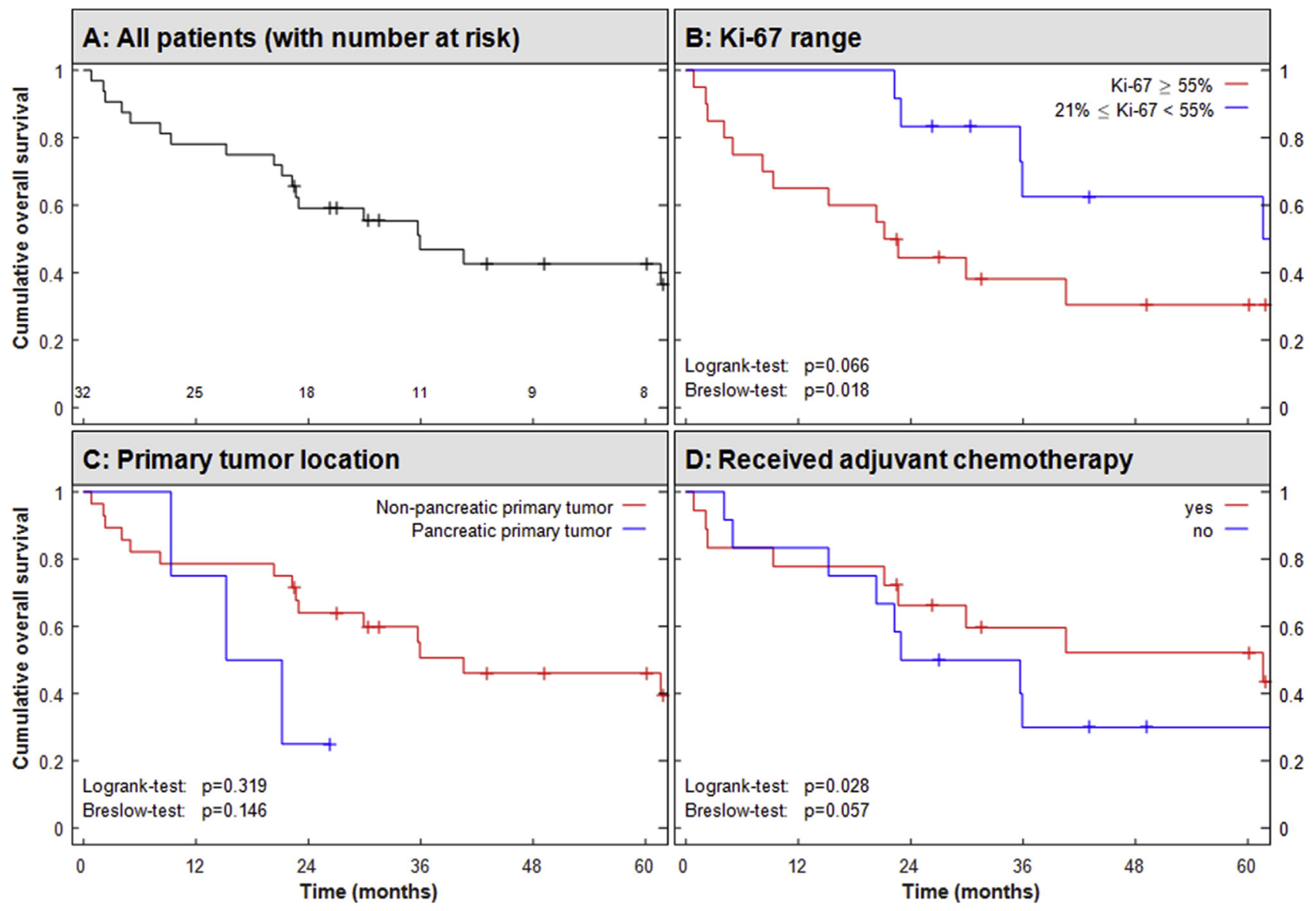

Figure 1. Overall survival after surgical treatment of liver metastases in patients with gastroenteropancreatic neuroendocrine carcinoma. Survival assessed by Kaplan-Meier and stratified by prognostic markers. Overall survival for the entire group treated with curative intent (A), stratified by Ki-67 $\geq 55 \%$ versus $<55 \%$ (B), stratified by pancreatic primary versus non-pancreatic primary (C), and stratified by received versus not received adjuvant chemotherapy (D). 
Table 2

Survival for 32 patients with surgically treated metastatic gastroenteropancreatic neuroendocrine carcinoma.

\begin{tabular}{|c|c|c|c|c|c|c|c|c|c|c|c|}
\hline & \multirow[t]{2}{*}{ No. Patients } & \multicolumn{3}{|c|}{ Overall survival (months) } & \multicolumn{2}{|l|}{ P-value } & \multicolumn{3}{|c|}{$\begin{array}{l}\text { Progression free survival } \\
\text { (months) }\end{array}$} & \multicolumn{2}{|l|}{ P-value } \\
\hline & & Median (CI) & 3-year & 5-year & Logrank & Breslow & Median (CI) & 3-year & 5-year & Logrank & Breslow \\
\hline All patients & 32 & $35.9(20.6-51.3)$ & $47 \%$ & $43 \%$ & & & $8.4(3.9-13)$ & $16 \%$ & $13 \%$ & & \\
\hline Ki-67 & & & & & 0.066 & 0.018 & & & & 0.162 & 0.074 \\
\hline$\geq 55 \%$ & 20 & $21.2(16.3-26.0)$ & $38 \%$ & $31 \%$ & & & $5.0(0.2-7.6)$ & $10 \%$ & $10 \%$ & & \\
\hline $21-54 \%$ & 12 & $61.6(21.8-101.5)$ & $63 \%$ & $63 \%$ & & & $11.3(8.1-14.4)$ & $25 \%$ & $17 \%$ & & \\
\hline Primary tumor location & & & & & 0.319 & 0.146 & & & & 0.382 & 0.122 \\
\hline Pancreatic primary tumor & 14 & $40.6(32.7-48.5)$ & $53 \%$ & $44 \%$ & & & $10(7.0-13.0)$ & $14 \%$ & $14 \%$ & & \\
\hline Non-pancreatic primary tumor & 18 & $21.2(15.7-26.7)$ & $44 \%$ & $44 \%$ & & & $3.8(0-8.0)$ & $17 \%$ & $11 \%$ & & \\
\hline Number of metastases & & & & & 0.451 & 0.536 & & & & 0.198 & 0.292 \\
\hline $0-2$ metastases & 18 & $61.6(11.1-112.2)$ & $60 \%$ & $52 \%$ & & & $10(6.3-13.6)$ & $22 \%$ & $17 \%$ & & \\
\hline$>2$ metastases & 12 & $23(2.25-43.7)$ & $30 \%$ & $30 \%$ & & & $6.3(2.3-10.1)$ & $8 \%$ & $8 \%$ & & \\
\hline Adjuvant chemotherapy & & & & & 0.028 & 0.057 & & & & 0.068 & 0.031 \\
\hline Yes & 13 & $66.8^{\mathrm{a}}$ & $69 \%$ & $69 \%$ & & & $11.5(7.5-15.4)$ & $23 \%$ & $23 \%$ & & \\
\hline No & 18 & $29.9(10-49,8)$ & $31 \%$ & $24 \%$ & & & $5.0(0-11,2)$ & $11 \%$ & $6 \%$ & & \\
\hline Neoadjuvant chemotherapy & & & & & 0.142 & 0.528 & & & & 0.148 & 0.259 \\
\hline Yes & 12 & $>29.9$ & $57 \%$ & $57 \%$ & & & $6.6(0-16.4)$ & $25 \%$ & $25 \%$ & & \\
\hline No & 20 & $35.7(14.1-57.2)$ & $41 \%$ & $34 \%$ & & & $8.4(0.35-16.5)$ & $10 \%$ & $5 \%$ & & \\
\hline $\begin{array}{l}\text { Complete macroscopic } \\
\text { resection or ablation }\end{array}$ & & & & & 0.299 & 0.363 & & & & 0.556 & 0.914 \\
\hline Yes & 28 & $35.7(17.6-53.8)$ & $43 \%$ & $38 \%$ & & & $8.4(2.0-14.8)$ & $18 \%$ & $14 \%$ & & \\
\hline No & 4 & $>30.5^{b}$ & $75 \%$ & $75 \%$ & & & $6.6(4.3-9.0)$ & $0 \%$ & $0 \%$ & & \\
\hline Differentiation & & & & & 0.9 & 0.542 & & & & 0.594 & 0.926 \\
\hline Well differentiated & 2 & $35.9^{c}$ & & & & & $6.0^{\mathrm{d}}$ & & & & \\
\hline Poorly differentiated & 24 & $35.7(13.5-57.9)$ & $48 \%$ & $42 \%$ & & & $8.4(4.0-12.8)$ & $17 \%$ & $13 \%$ & & \\
\hline Radiofrequency ablation $\left(\right.$ RFA) ${ }^{\mathrm{e}}$ & & & & & 0.084 & 0.374 & & & & 0.430 & 0.885 \\
\hline Yes & 9 & $29.9(20.1-39.7)$ & $0 \%$ & $0 \%$ & & & $8.4(1.3-15.5)$ & $0 \%$ & $0 \%$ & & \\
\hline No & 22 & $61.6(27.7-95.6)$ & $64 \%$ & $58 \%$ & & & $8.7(4.5-12.9)$ & $23 \%$ & $18 \%$ & & \\
\hline
\end{tabular}

Numbers in bold reflect significant differences in outcomes.

a Not computable.

b One died after 15.2 months, three still alive followed for 31 months, 43 months and 49 months.

c One died after 35.9 months. One is still alive after 22.6 months.

d One had recurrence after 6 months, one after 11.3 months.

e Six patients with isolated RFA, three patients with combined resection/RFA.

of 22.6 months (still alive) and 35.9 months (dead). Table 2 presents OS for the entire material and subgroups.

\section{Recurrence and progression free survival}

Of the 32 patients, 28 had recurrence (five-year PFS: 13\%) (Fig. 2a). Three had local recurrence, eighteen had hepatic recurrence, eight had lymph node recurrence, three had pulmonary recurrence, one had bone recurrence and five had recurrence in other localizations (ovary, cutaneous, peritoneum). Four of the patients had simultaneous recurrence in several locations. Twenty-three of the recurrences were treated with chemotherapy, five with surgery, two with best supportive care and five with other modalities (liver embolization, everolimus, interferon, somatostatin analog). The median progression-free survival after liver surgery was 8.4 months (95\% CI 3.9-13) (Fig. 2a). In the group of patients with a Ki-67 of $21-54 \%$ there was a trend towards longer PFS than in the group of patients with Ki67 $\geq 55 \%$ (11.3 vs. 5.0 months $\mathrm{P}=0.074$ ) (Fig. 2b). Having a pancreatic primary did not significantly affect PFS (40.6 vs. 21.2 months $\mathrm{P}=0.146$ ) (Fig. 2c). Receiving adjuvant chemotherapy significantly improved PFS (11.5 vs
5.0 months $\mathrm{P}=0.031$ ) (Fig. 2d). Table 2 presents PFS for the entire material and subgroups.

\section{Postoperative complications after liver surgery}

There were no fatalities due to surgery. However, eleven patients had one or more postoperative complications: wound infection, abscess in the cicatrice, pancreatitis, pneumonia, pancreatic leakage, small bowel obstruction, portal vein thrombosis, pleural effusion and need for parenteral nutrition. The most serious adverse event was a postoperative liver failure occurring due to only $17 \%$ remaining liver tissue after resection of a $22 \mathrm{~cm}$ large liver metastasis. This patient received a liver transplant one week after liver resection and lived for 5 years before he died of NEC. Table 3 classifies the postoperative complications according to the Clavien-Dindo classification.

\section{Characteristics of long-term survivors without recurrence}

The four patients who were free from disease at the time of analysis had been followed for a median of 72.5 months 


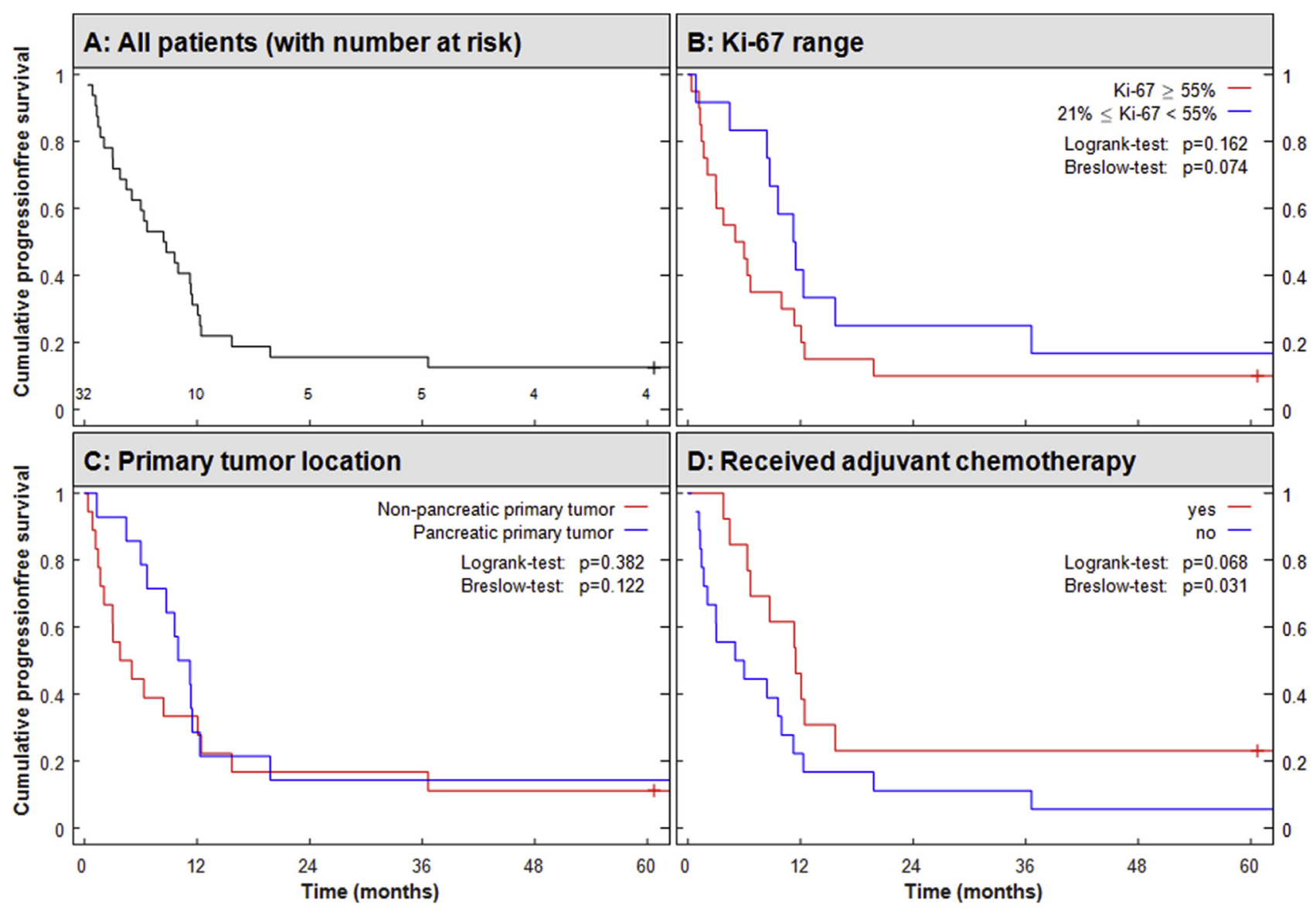

Figure 2. Progression free survival after surgical treatment of liver metastases in patients with gastroenteropancreatic neuroendocrine carcinoma. Survival assessed by Kaplan-Meier and stratified by prognostic markers. Progression free survival for the entire group treated with curative intent (A), stratified by Ki-67 $\geq 55 \%$ versus $<55 \%$ (B), stratified by pancreatic primary versus non-pancreatic primary (C), and stratified by received versus not received adjuvant chemotherapy (D).

(range 60-184 months). Two had a primary pancreatic NEC, one had a colonic NEC and one had a rectal NEC. All had non-small cell morphology, three were poorly differentiated and one had an unknown tumor

Table 3

Postoperative complications after liver surgery in 32 patients with metastatic gastroenteropancreatic neuroendocrine carcinoma.

\begin{tabular}{ll}
\hline $\begin{array}{l}\text { Postoperative complications } \\
\text { according to the Clavien-Dindo }\end{array}$ & Number of patients \\
\hline Grade I & 2 \\
- Wound infection & \\
Grade II & 1 \\
- Pneumonia & 1 \\
- Peritonitis & 1 \\
- Abscess in the cicatrice & 1 \\
- Total parenteral nutrition & 1 \\
- Portal vein thrombosis & \\
Grade III & 1 \\
- Pancreatitis & 1 \\
- Pancreatic leakage & 1 \\
- Pleural effusion & 1 \\
- Bowel obstruction & \\
Grade IVa & 1 \\
- Liver failure &
\end{tabular}

differentiation. The Ki-67 was $30 \%, 70 \%, 90 \%$ and $90 \%$. The patients had few metastases (1-4) with a unilateral, synchronous presentation. The median size of the largest metastasis was $5.3 \mathrm{~cm}$ (range $3-20 \mathrm{~cm}$ ). All resections were R0. One patient received both neoadjuvant and adjuvant cisplatin/etoposide, one patient did not receive any chemotherapy and for the last two patients one received neoadjuvant and one received adjuvant cisplatin/etoposide.

\section{Discussion}

As GEP-NEC is an aggressive disease with a median survival of 11 months and 3-year survival of less than $10 \%$ after palliative chemotherapy, it is important to explore alternative methods that could improve survival. ${ }^{11}$ The described 32 patients with liver surgery presented with good performance status and most had liver surgery close to the time of diagnosis, reflecting a low tumor burden. The median OS of 35.9 months and 5-year survival of $43 \%$ is surprisingly high and in contrast to results from previous studies. Saxene and colleagues found a median OS of 15 months for eleven patients with high grade NEC. ${ }^{16}$ However, their inclusion criteria and methods differ from 
ours in several aspects. The high grade NEC patients represented only a small fraction of their material (11/74) and the surgery was not exclusively performed with a curative intent, but in 35\% also with a debulking purpose. Furthermore, patient characteristics regarding tumor load, tumor grade (Ki-67-level), tumor differentiation and primary site were not specified for the high-grade group. Cho and colleagues did a similar study on metastatic NEN patients including seven high-grade cases. ${ }^{15}$ These patients survived for a median of 6 months, but again relevant patient characteristics are not described.

Recent studies have demonstrated a new subgroup of neuroendocrine neoplasms with predominantly pancreatic primaries and a high proliferation rate (Ki $67>20 \%$ ), which place them into the G3 category, but with histologically well-differentiated morphology, thus coined as NET G3. ${ }^{22,23}$ However, the distinction between NET G3 and NEC may be difficult to establish on routine pathologic assessment. ${ }^{24,25}$ Survival for the NET-G3 subgroup appears to be significantly longer than for patients with poorly differentiated high-grade tumors. ${ }^{26}$ To ascertain that our material did not exclusively consist of well-differentiated NET G3 cases, four experienced neuroendocrine pathologists reexamined available tumor slides. Only two of twenty-six patients had verified morphological well-differentiated tumors, and this can therefore not explain the long survival in our patients. In addition, given the higher incidence of NET G3 in pancreatic primaries we compared pancreatic primaries with the non-pancreatic primaries. This did not reveal a significant difference in overall survival and progression-free survival in this small sample size. Higher proliferation rate in GEP-NEC is associated with significantly worse OS. ${ }^{11,27,28}$ In the Nordic NEC study, median OS was 14 months and 10 months for chemotherapytreated patients with $\mathrm{Ki} 67: 21-55 \%$ and $\mathrm{Ki} 67 \geq 55 \%$ respectively. ${ }^{11}$ When we stratified our patients according to Ki-67 level, the group with $\mathrm{Ki}-67: 21-54 \%$ had the longest PFS and OS. However, also patients with a higher $\mathrm{Ki}-67$ seemed to benefit from surgical treatment of liver metastases, with a median OS of 21.2 months and a 5-year survival of $31 \%$. Our results seem to indicate a benefit from receiving adjuvant chemotherapy, however, the patients received a variety of regimens and specific recommendations are therefore hard to make. Of special interest are the four patients without disease recurrence after a median follow-up of 72 months. These patients seem to have been cured of their disease. Unfortunately we could not identify specific patient characteristics or treatment differences that could explain the long term PFS for these four patients. The possible benefit of surgical treatment must be weighed against the risk of postoperative complications and of rapid postoperative disease progression. In our cohort there were no fatalities due to surgery, however the postoperative complication rate was $22 \%$.

Our study has some strengths and limitations. All patients are recruited from high-volume NEN centers reporting data to a central Nordic GEP-NEC database. Therefore the patients are well characterized with clinical and histopathological evaluation and with follow-up evaluated by NEN experts. However, a limitation is the retrospective design with a risk of confounding factors. The patients receiving surgical treatment of the liver metastases are probably highly selected with good performance status, low comorbidity, no progression during preoperative chemotherapy and limited disease. Any comparison with the surgically untreated liver-only metastatic patients is therefore difficult. The criteria for liver surgery and neoadjuvant/adjuvant chemotherapy depended on the local expertise and were thus not consistent throughout this cohort. In addition information regarding follow-up visits and imaging performed is limited.

In conclusion, curatively intended surgical treatment of liver metastases in selected GEP-NEC patients may be considered as a part of the treatment plan, especially if the Ki-67 is in the relatively lower G3 range. Further prospective studies would be necessary to define clinical and pathologic criteria for this treatment modality.

\section{Conflict of interest statement}

H Sorbye has received grants from the Nordic Cancer Union, The Norwegian Cancer Society, Ipsen, Novartis and Eckbo Foundations regarding the NEC registries. A Perren was supported by the Swiss National Science Foundation (SNF 310030_144236). ET Janson has received grants from the Swedish Cancer Society and Lions Fund for Cancer Research. For the remaining authors none were declared.

\section{Acknowledgements}

We thank Hege Elvebakken (Department of Oncology, Aalesund Hospital Norway) for recruitment of one patient. Jean-Yves Scoazec (Department of Pathology, Gustave Roussy Institute, Villejuif, France) and Birgitte Federspiel (Department of Pathology, Rigshospitalet, University of Copenhagen, Denmark) assisted the pathological reviewing process. H Sorbye has received grants from the Nordic Cancer Union, The Norwegian Cancer Society, Ipsen, Novartis and Eckbo Foundations regarding the NEC registries. A Perren was supported by the Swiss National Science Foundation (SNF 310030_144236). E T Janson has received grants from the Swedish Cancer Society and Lions Fund for Cancer Research.

\section{References}

1. Bosman FT, World Health Organization International Agency for Research on Cancer. WHO classification of tumours of the digestive system. 4th ed. Lyon: International Agency for Research on Cancer; 2010. p. 417. 
2. Heetfeld M, Chougnet CN, Olsen IH, et al. Characteristics and treatment of patients with G3 gastroenteropancreatic neuroendocrine neoplasms. Endocr Relat Cancer 2015;22(4):657-64.

3. Ilett EE, Langer SW, Olsen IH, Federspiel B, Kjaer A, Knigge U. Neuroendocrine carcinomas of the gastroenteropancreatic system: a comprehensive review. Diagnostics (Basel) 2015;5(2):119-76.

4. Korse CM, Taal BG, van Velthuysen ML, Visser O. Incidence and survival of neuroendocrine tumours in The Netherlands according to histological grade: experience of two decades of cancer registry. Eur $J$ Cancer 2013;49(8):1975-83.

5. Smith JD, Reidy DL, Goodman KA, Shia J, Nash GM. A retrospective review of 126 high-grade neuroendocrine carcinomas of the colon and rectum. Ann Surg Oncol 2014;21(9):2956-62.

6. Sorbye H, Strosberg J, Baudin E, Klimstra DS, Yao JC. Gastroenteropancreatic high-grade neuroendocrine carcinoma. Cancer 2014; 120(18):2814-23.

7. Yamaguchi T, Machida N, Morizane C, et al. Multicenter retrospective analysis of systemic chemotherapy for advanced neuroendocrine carcinoma of the digestive system. Cancer Sci 2014;105(9):1176-81.

8. Janson ET, Sorbye H, Welin S, et al. Nordic guidelines 2014 for diagnosis and treatment of gastroenteropancreatic neuroendocrine neoplasms. Acta Oncol 2014;53(10):1284-97.

9. Kunz PL, Reidy-Lagunes D, Anthony LB, et al. Consensus guidelines for the management and treatment of neuroendocrine tumors. Pancreas 2013;42(4):557-77.

10. Pavel M, Baudin E, Couvelard A, et al. ENETS Consensus Guidelines for the management of patients with liver and other distant metastases from neuroendocrine neoplasms of foregut, midgut, hindgut, and unknown primary. Neuroendocrinology 2012;95(2):157-76.

11. Sorbye H, Welin S, Langer SW, et al. Predictive and prognostic factors for treatment and survival in 305 patients with advanced gastrointestinal neuroendocrine carcinoma (WHO G3): the NORDIC NEC study. Ann Oncol Off J Eur Soc Med Oncol ESMO 2013;24(1):152-60.

12. Garcia-Carbonero R, Sorbye H, Baudin E, et al. ENETS consensus guidelines for high-grade gastroenteropancreatic neuroendocrine tumors and neuroendocrine carcinomas. Neuroendocrinology 2016; 103(2): 186-94.

13. Oberg K, Knigge U, Kwekkeboom D, Perren A, Group EGW. Neuroendocrine gastro-entero-pancreatic tumors: ESMO Clinical Practice Guidelines for diagnosis, treatment and follow-up. Ann Oncol Off $J$ Eur Soc Med Oncol ESMO 2012;23(Suppl 7):vii124-30.

14. Strosberg JR, Coppola D, Klimstra DS, et al. The NANETS consensus guidelines for the diagnosis and management of poorly differentiated (high-grade) extrapulmonary neuroendocrine carcinomas. Pancreas 2010;39(6):799-800.

15. Cho CS, Labow DM, Tang L, et al. Histologic grade is correlated with outcome after resection of hepatic neuroendocrine neoplasms. Cancer 2008;113(1):126-34.
16. Saxena A, Chua TC, Sarkar A, et al. Progression and survival results after radical hepatic metastasectomy of indolent advanced neuroendocrine neoplasms (NENs) supports an aggressive surgical approach. Surgery 2011;149(2):209-20.

17. Sorbye H, Westre B, Horn A. Curative surgery after neoadjuvant chemotherapy in metastatic poorly differentiated neuroendocrine carcinoma. Eur J Surg Oncol J Eur Soc Surg Oncol Br Assoc Surg Oncol 2007;33(10):1209-10.

18. Power DG, Asmis TR, Tang LH, Brown K, Kemeny NE. High-grade neuroendocrine carcinoma of the colon, long-term survival in advanced disease. Med Oncol 2011;28(Suppl 1):S169-74.

19. Tanis E, Nordlinger B, Mauer M, et al. Local recurrence rates after radiofrequency ablation or resection of colorectal liver metastases. Analysis of the European Organisation for Research and Treatment of Cancer \#40004 and \#40983. Eur J Cancer 2014;50(5):912-9.

20. Wei AC, Greig PD, Grant D, Taylor B, Langer B, Gallinger S. Survival after hepatic resection for colorectal metastases: a 10-year experience. Ann Surg Oncol 2006;13(5):668-76.

21. Rees M, Tekkis PP, Welsh FK, O'Rourke T, John TG. Evaluation of long-term survival after hepatic resection for metastatic colorectal cancer: a multifactorial model of 929 patients. Ann Surg 2008; 247(1):125-35.

22. Basturk O, Tang L, Hruban RH, et al. Poorly differentiated neuroendocrine carcinomas of the pancreas: a clinicopathologic analysis of 44 cases. Am J Surg Pathol 2014;38(4):437-47.

23. Velayoudom-Cephise FL, Duvillard P, Foucan L, et al. Are G3 ENETS neuroendocrine neoplasms heterogeneous? Endocr Relat Cancer 2013;20(5):649-57.

24. Tang LH, Basturk O, Sue JJ, Klimstra DS. A practical approach to the classification of WHO Grade 3 (G3) Well-differentiated Neuroendocrine Tumor (WD-NET) and Poorly Differentiated Neuroendocrine Carcinoma (PD-NEC) of the pancreas. Am J Surg Pathol 2016; 40(9):1192-202.

25. Tang LH, Untch BR, Reidy DL, et al. Well-differentiated neuroendocrine tumors with a morphologically apparent high-grade component: a pathway distinct from poorly differentiated neuroendocrine carcinomas. Clin Cancer Res 2016;22(4):1011-7.

26. Basturk O, Yang Z, Tang LH, et al. The high-grade (WHO G3) pancreatic neuroendocrine tumor category is morphologically and biologically heterogenous and includes both well differentiated and poorly differentiated neoplasms. Am J Surg Pathol 2015; 39(5):683-90.

27. Olsen IH, Sorensen JB, Federspiel B, et al. Temozolomide as second or third line treatment of patients with neuroendocrine carcinomas. SciWorldJ 2012;2012:170496.

28. Hadoux J, Malka D, Planchard D, et al. Post-first-line FOLFOX chemotherapy for grade 3 neuroendocrine carcinoma. Endocr Relat cancer 2015;22(3):289-98. 